Copyright (C) 2006 IEEE. Reprinted from

IEEE International Conference on Mechatronics and Automation

(2006 : Luoyang, China)

This material is posted here with permission of the IEEE. Such permission of the IEEE does not in any way imply IEEE endorsement of any of the University of Adelaide's products or services. Internal or personal use of this material is permitted. However, permission to reprint/republish this material for advertising or promotional purposes or for creating new collective works for resale or redistribution must be obtained from the IEEE by writing to pubs-permissions@ieee.org.

By choosing to view this document, you agree to all provisions of the copyright laws protecting it. 


\title{
Novel Wind Sensor for Robotic Chemical Plume Tracking
}

\author{
Tien-Fu Lu and Chee Liang Ng \\ School of Mechanical Engineering, University of Adelaide, Australia \\ tien-fu.lu@adelaide.edu.au
}

\begin{abstract}
Wind sensors are widely used to measure wind speed and direction for such as weather forecasting or for other purposes including measuring wind flow of wind tunnel in a laboratory. Due to its wide applications, many wind sensors are commercially available off-the-shelf. This study concerns wind direction and speed sensing for robotic chemical plume tracking studies and applications. Therefore, it is highly demanded to have a wind sensor that has smaller size, lighter weight and less power consumption. In this paper, from the design of the wind sensor down to the postprocessing of the sensor reading data are presented. The result shows that the wind sensor performance is satisfactory and can be implemented for robotic chemical plume tracking study to provide proper wind speed and direction information.
\end{abstract}

Index Terms - wind sensor, chemical plume tracking, robotic, wind speed, wind velocity

\section{INTRODUCTION}

Insects use wind and chemical sensing to perform a number of tasks. The combination of information obtained about the wind velocity, wind direction and airborne chemical concentrations enable insects to accurately locate food, prey and mates. Research aimed to understand the mechanisms happening within the insects and construct the robotic models can provide benefits for not only biologists to expand knowledge in this area but also engineers to create practical applications. The applications include more efficient use of biological pest control and operations in dangerous or difficult environments such as high concentrations of poison gas and nuclear radiation.

There have been a number of studies concentrating on insect plume tracking behaviours and the associated robotic modelling in constrained and open environments. For example, for single point sensing plume tracking behaviour, pure anemotaxi was the simplest implemented algorithm. Two algorithms implementing anemotaxi were presented by Balkovsky et al that involve searching an area upwind of the point of odour contact. [1]. An upwind zigzagging, often referred to as conunterturning, was identified by several researchers [2].

To study insect plume tracking behaviours and/or mimicking the design and behaviour of insects, several robotic models have been developed. For example, Andrew et al [3] developed a chemical sensing robot for the comparison of some robot chemotaxi algorithms. Yoshihiko Kuwana and Isao Shimoyama presented a pheromone-guided robot synthesizing a small robot and a biosensor that is the real antennae from a silkworm moth [4]. David Harvey [6] constructed a small mobile insect robot for the purpose of verifying several hypotheses as an explanation for observed plume tracking behaviours.

These algorithms and robotic models have all considered the combined effects of chemical sensing with windfollowing to produce chemical plume tracking behaviours. Therefore, the development of appropriate wind and chemical sensors is apparently essential that are to be incorporated into robotic models for insect chemical plume tracking studies and related robotic applications. As a result, there have been a number of developments and studies in the area. Some good and robust sensors have also been developed and commercially available. However, since this paper focuses on wind sensing, only some wind sensing related studies are reviewed.

Regarding wind sensing, Lomas [7] used the temperature changes of hot-wire anemometers caused by convection of the wind flowing over the heated wire and reflected by a change in its resistance to measure fluid velocity. Bruun [8] measured the time of flight of a small volume of heated air produced by pulsing a voltage to temporarily heat a wire. The time and direction of flight of this heated air is measured by sensor wires surrounding the heater and this arrangement is referred as pulsed wire anemometer. Hayes [9] and Ishida [10] applied Shibaura thermal anemometer (F6201-1) for their mobile robots. The sensor produces a voltage signal corresponding to the velocity of the wind, with a logarithmic relationship between the wind speed and voltage output. This relationship is almost linear at low velocities, particularly for the range of 0 to $20 \mathrm{~cm} /$ second [11]. However, this sensor takes one second to respond to a velocity change, which is considered too slow for the presented study. Chapman et al. presented a wind sensor based on the cercal filiform hairs of the cricket. The sensor was used in a group of four. It was able to locate a wind source of less than $1 \mathrm{~m} / \mathrm{s}$ but can not determine wind speed and accurate wind direction.

Even though a wide range of wind sensors have been so far developed to measure wind direction and velocity, most of them are for outdoor use. They either can not measure low airflows as required for insect plume tracking studies or too slow in responding to changes. To 
address this, Russell and Kennedy [5] constructed a novel wind sensor for a miniature mobile robot using the changes in velocity of a small paddle rotated by a DC motor to measure both the velocity and direction of the wind flow. However, as discussed by David Harvey [6], it has a number of disadvantages including the inherent inaccuracy in its measurement device, the optical encoder, and reduction of accuracy through approximations of the physics of the situation. Alternatively, using ultrasonic sensors to measure wind velocity and direction has no lower threshold speed [12]. However, commercially produced units all required the transmitter and receiver to be apart at least $150 \mathrm{~mm}$ and cost over AUD\$2000. David Harvey et al. [13] presented a wind sensor for the wind range of $0.3 \mathrm{~m} / \mathrm{s}$ to $1 \mathrm{~m} / \mathrm{s}$ using thermistor based sensor (F6201-1, Shibaura Electronics Co., Japan). Even through it can measure low wind velocity and consumes low power with no moving parts but it is relatively bulky and could be too big for most miniature robots.

To address the needs of measuring low wind speed with smaller sensor size, lighter weight and faster response, this paper presents the design and investigation of a novel new wind sensor.

\section{WIND SENSOR DESIGN}

In addition to measure air flow at speeds of $1 \mathrm{~m} / \mathrm{s}$ and lower, the wind sensor design is also aimed to be small in size, light in weight, low power consumption, low computational overhead, robust and low in cost. It is intended that this sensor will be later implemented on a miniature robot for chemical plume tracking related studies.

The design and photo of the proposed wind sensor is shown in Figure 1 below. The circular rod at the top of wind sensor is changeable. Material used in the current design is light weight foam. Larger rod length and cross section area will provide larger surface to maximise the drag that is directly corresponded to the wind flow. This is however limited by the size constraint of the sensor for practical usage. Symmetric shape makes sure the same drag will still be there when wind direction changes.

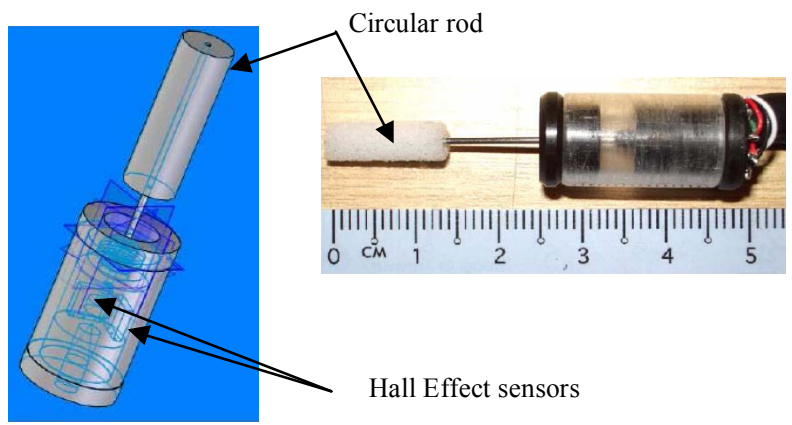

Figure 1: The isometric view and photo of the wind sensor design
At the other end of the central bar, there is a small permanent magnet attached as illustrated in Figure 2. A pivot point is designed and its rough location is indicated in Figure 2. As a result, the magnet at the end of the bar will therefore swing in the opposite direction of the wind and therefore gets closer to or further away from the two Hall Effect sensors near by as illustrated in Figure 3. This will vary the density of the magnetic flux exerted on Hall Effect sensors and therefore the output voltage from these two sensors will vary accordingly. This variation can then be used to potentially determine the wind velocity and direction. This design gives instantaneous response of sensor readings to wind direction and/or velocity changes. There is a transparent casing encloses the small magnet and Hall Effect sensors to prevent them from being directly effected by wind blow.

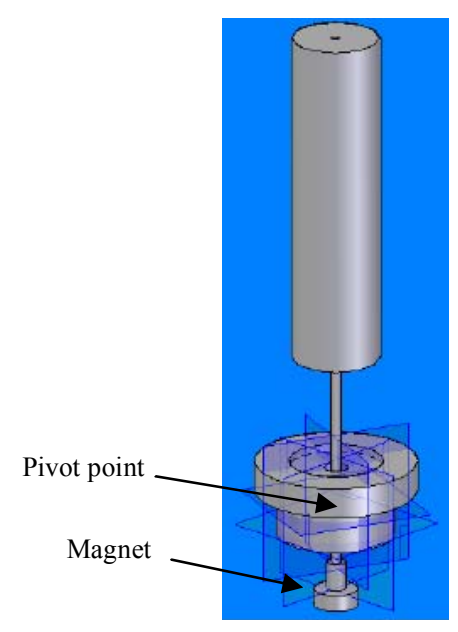

Figure 2: Moving part of the wind sensor

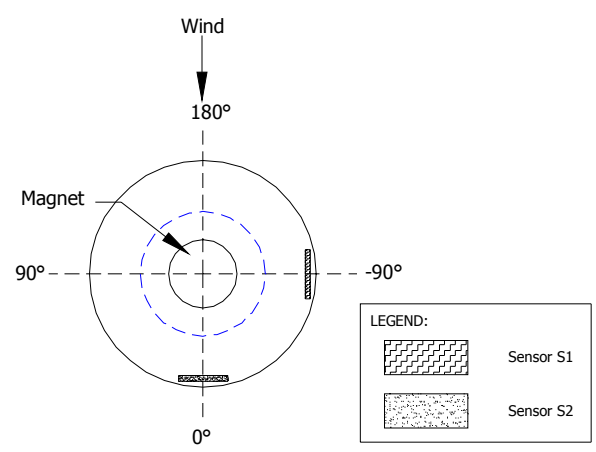

Figure 3: The arrangement of two Hall Effect sensors [14]

\section{EXPERIMENTAL SETUP}

The purposes of the experiments are to determine the limitations of the wind sensor design and provide analysis for future design improvement. Two different wind tunnels were used for the experiments as shown in Figure 
4. The large wind tunnel was used for the generation of higher wind speeds (above $0.7 \mathrm{~m} / \mathrm{s}$ ) and the small wind tunnel is responsible for the generation of lower wind speeds (below $0.7 \mathrm{~m} / \mathrm{s}$ ).
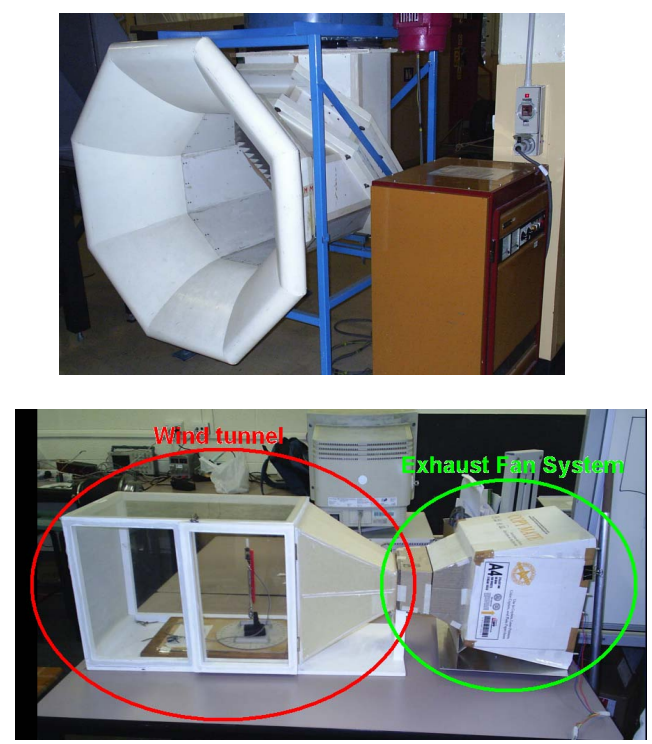

Figure 4: Large (top) and small (bottom) wind tunnels

Rather than changing the wind direction directly that is technically more difficult, the wind sensor was rotated with an angle plate that has $5^{\circ}$ increments and a stand for the sensor to be vertically attached to and situated at the centre of the angle plate as shown in Figure 5. Therefore, relatively the wind direction can be varied. Anemometer was used to measure and make sure the small and large wind tunnels do produce the required wind speeds before experiments were preceded.

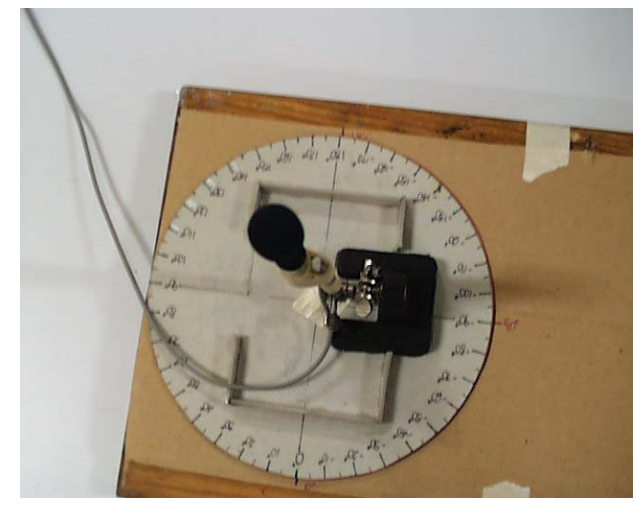

Figure 5: Angle plate and sensor

The voltage output of the two Hall Effect sensors was registered by a digital multimeter and sampled by a computer via the serial port. Five readings were taken and averaged for each wind direction. The wind flow direction was changed by rotating the sensor attached to the stand on the angle plate with $10^{\circ}$ increments clockwise. New readings will only be taken after the angle plate is rotated and waited for 10 seconds to allow the disturbance caused by human intervention settled. This procedure was repeated for all wind speeds to be tested ranging from 0.3 $\mathrm{m} / \mathrm{s}$ to $1.0 \mathrm{~m} / \mathrm{s}$.

\section{RESULTS AND DISCUSSIONS}

The voltage outputs of sensors 1 and 2 taken at wind speed ranging from $0.3 \mathrm{~m} / \mathrm{s}$ to $1.0 \mathrm{~m} / \mathrm{s}$ for all $360^{\circ}$ with $10^{\circ}$ increments are plotted in Figure 6.

Eight sets of $6^{\text {th }}$ order polynomial equations were created to fit these data sets. These equations are listed in Appendix A. Figure 7 shows the plots of the original data together with the lines generated by the fitted polynomial equations. In those equations listed in Appendix A, Y1 represents the voltage output of sensor 1, Y2 represents the voltage output of sensor 2 and $\mathrm{X}$ is the wind direction in degrees from $0^{\circ}$ to $360^{\circ}$.

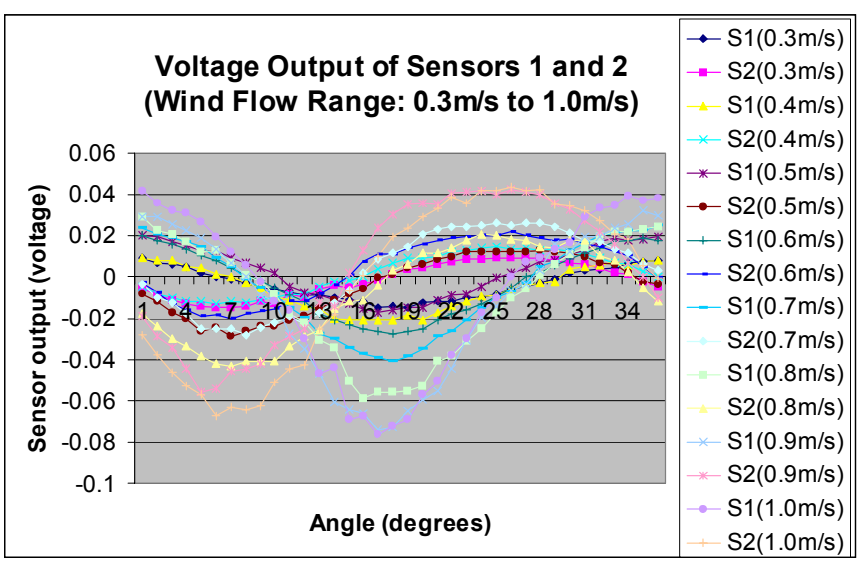

Figure 6: Voltage output of sensors 1 and 2

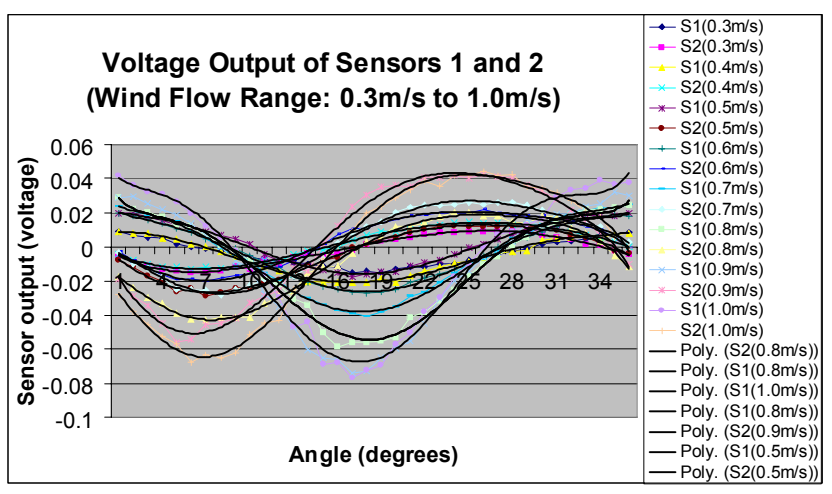

Figure 7: Approximated sensor output using polynomial equations

When the wind sensor was implemented in experiments, sets of $\mathrm{Y} 1$ and $\mathrm{Y} 2$ readings were obtained. Once one set of reading was sampled, this set of reading (Y1 and Y2) was 
then used to solve the 8 sets of $6^{\text {th }}$ order polynomial equations. One of the 8 sets of equations would produce the same or very close values for $\mathrm{X}$, which is the wind direction. This therefore determines the wind direction. At the same time, this specific set of equations also gives the wind speed as each set of equations is associated with a specific wind speed.

For instance, a set of readings was obtained when the sensor was oriented $10^{\circ}$ and under $0.3 \mathrm{~m} / \mathrm{s}$ wind velocity. The reading for Sensor 1 was 0.009 volt and for Sensor 2 was -0.005 volt. By solving the 8 sets of equations, it was found that the set of equations associated with wind speed $0.3 \mathrm{~m} / \mathrm{s}$ produced the closest values of $\mathrm{X}$ among all 8 sets of equations, which were $9.15^{\circ}$ and $9.89^{\circ}$ with averaged value of $9.52^{\circ}$. This result shows the presented sensor works but with a small error, which in this case is $4.8 \%$ and could be acceptable for robotic chemical plume tracking applications. This is because the exact wind direction and velocity are not required for the robot to figure out how to turn and move.

It can be seen from Figure 7 that the sensor readings have the shape of a sine and a cosine curves. However, the data do not match the sine and cosine curves nicely in this collected data set. The computational efficiency for solving the wind direction and velocity will become very good if the since and cosine curves could fit the data set nicely. It is therefore important and worthwhile for further investigation. It maybe worthwhile to double check several aspects, such as double checking experimental setups, repeating the experiments to collect other sets of data to see if the data will match with sine and cosine curves better, and making sure two Hall Effect sensors are positioned accurately.

As only few tests were performed in this presented study, there is a need to future test the wind sensor under various combinations of wind direction and velocity to better evaluate the performance of the sensor. With more tests, the maximum, minimum and average errors for wind direction and velocity sensing can be statistically derived.

There is no damping effect incorporated in the current sensor design, the readings of sensors respond to even very tiny wind direction or velocity changes instantaneously and result in unwanted noises caused by the swinging effects of the magnet. This is currently dealt with by averaging several readings. However, the design of the wind sensor could be improved to incorporate damping effects in the future.

\section{CONCLUSIONS AND FUTURE WORK}

A new novel wind sensor is presented in the paper for the purpose of robotic chemical plume tracking studies and related applications. It is demonstrated that the sensor meets the goals and works with satisfactory performance. However, currently it is necessary to solve 8 sets of $6^{\text {th }}$ order polynomial equations every time once a set of readings from sensors 1 and 2 are taken to figure out a wind speed and direction. This demonstrates the need to further investigate the way of post-processing the data with the hope of reducing the computational expenses, which is under investigation at the moment. Besides, to further reduce the overall sensor size and weight is also under the agenda of the future work.

There is a non-linear relationship between the value of the magnetic flux and the air gap. This is however not addressed and studied in this paper and can be further investigated in the future.

\section{ACKNOWLEDGEMENT}

The authors would like to greatly acknowledge the support from the Electroncis Workshop at the School of Mechanical Engineering, University of Adelaide in fabrication the sensor and giving ideas. Besides, some ground work done by Mr. Wilson Handoko and Mr. Fei Yong are much appreciated.

\section{REFERENCES}

[1] E. Balkovsky and B. I. Shraiman, "Olfactory search at high Reynolds number," Proceedings of the National Academy of Sciences (USA), 99(20):12589-12593, October 2002.

[2] T.C. Baker. Mechanisms in Insect Olfaction, chapter Pheromone-modulated movements of flying moths, pages 39-48. Clarendon Press, Oxford, 1986.

[3] R. Andrew Russel, Alireza Bab-Hadiashar, Rod L. Shepherd, Gordon G. Wallace, "A comparison of reactive robot chemotaxis algorithms," Robotics and Autonomous Systems 45 (2003) 83-97

[4] Yoshihiko Kuwana, Sumito Nagasawa, Isao Shimoyama, Ryohei Kanzaki, "Synthesis of the pheromone-oriented behaviour of silkworm moths by a mobile robot with moth antennae as pheromone sensors," Biosensors \& Bioelectronics 14 (1999) 195202

[5] R.A Russell and S. Kennedy, "A novel airflow sensor for miniature mobile robot," Mechatronics, (10): 935942,2000

[6] Davey Harvey, "A robotic model wasp to investigate parasitoid insect plume tracking behaviour," internal report, Mechanical Engineering, University of Adelaide, May, 2003

[7] C.G. Lomas, Fundamentals of Hot Wire Anemometry, Cambridge University Press, 1986

[8] H.H. Bruun, Hot-Wire Anemometry: Principles and Signal Analysis, Oxford Science Publications, Oxford University Press, Oxford, 1995. 
[9] A.T. Hayes, A. Martinoli, and R.M. Goodman, "Swarm robotic odor source location", IEEE Sensors, June 2000

[10] H. Ishida, T. Suetsugu, T. Nakamoto, and T. Moriizumi, "Study of autonomous mobile sensing system for localistion of odor source using gas sensors and anemometric sensors," Sensors and Actuators A, (45): 153-157, 1994

[11] Shibaura Inc. F6201-1 specifications. 2002 May

[12] F.J. Gouveia and R.L. Baskett, "Evaluation of a new sonic anemometer for routine monitoring and emergency response applications," Technical report, Lawrence Livermore National Laboratory, February, 1997.

[13] Harvey, D.; Lu, Tien-Fu.; Keller, M., "Wind Sensor and Robotic Model Wasp Development," Australasian Conference on Robotics \& Automation 2003, 1-3 December 2003, Brisbane, Queensland, Australia

[14] Yong Fei Ming, "Experiments on Wind Sensor," internal report, July 2003, School of Mechanical Engineering, University of Adelaide

APPENDIX A: APPROXIMATED POLYNOMIAL EQUATIONS FOR SENSOR READINGS

\section{Equations for wind floe 0.3m/s (Equation Set 1):}

$Y_{1}=3 \mathrm{E}-16\left(X^{6}\right)-4 \mathrm{E}-13\left(X^{5}\right)+2 \mathrm{E}-10\left(X^{4}\right)-3 \mathrm{E}-$

$08\left(X^{3}\right)+2 \mathrm{E}-06\left(X^{2}\right)-0.0003(X)+0.0116$

$Y_{2}=3 \mathrm{E}-16\left(X^{6}\right)-4 \mathrm{E}-13\left(X^{5}\right)+2 \mathrm{E}-10\left(X^{4}\right)-5 \mathrm{E}-$

$08\left(X^{3}\right)+8 \mathrm{E}-06\left(X^{2}\right)-0.0006(X)+0.0002$;

Equations for wind floe $0.4 \mathrm{~m} / \mathrm{s}$ (Equation Set 2):

$Y_{1}=7 \mathrm{E}-16\left(X^{6}\right)-7 \mathrm{E}-13\left(X^{5}\right)+2 \mathrm{E}-10\left(X^{4}\right)-6 \mathrm{E}-$

$09\left(X^{3}\right)-3 \mathrm{E}-06\left(X^{2}\right)+9 \mathrm{E}-05(X)+0.008$

$Y_{2}=-2 \mathrm{E}-16\left(X^{6}\right)+3 \mathrm{E}-13\left(X^{5}\right)-1 \mathrm{E}-10\left(X^{4}\right)+1 \mathrm{E}-$

$08\left(X^{3}\right)+2 \mathrm{E}-06\left(X^{2}\right)-0.0003(X)-0.002$;

Equations for wind floe $0.5 \mathrm{~m} / \mathrm{s}$ (Equation Set 3):

$Y_{1}=1 \mathrm{E}-15\left(X^{6}\right)-1 \mathrm{E}-12\left(X^{5}\right)+5 \mathrm{E}-10\left(X^{4}\right)-6 \mathrm{E}-$

$08\left(X^{3}\right)+2 \mathrm{E}-06\left(X^{2}\right)-0.0001(X)+0.021$;
$Y_{2}=-1 \mathrm{E}-15\left(X^{6}\right)+1 \mathrm{E}-12\left(X^{5}\right)-4 \mathrm{E}-10\left(X^{4}\right)+6 \mathrm{E}-$

$08\left(X^{3}\right)+2 \mathrm{E}-06\left(X^{2}\right)-0.0006(X)-0.0008$;

Equations for wind floe $0.6 \mathrm{~m} / \mathrm{s}$ (Equation Set 4):

$Y_{1}=2 \mathrm{E}-15\left(X^{6}\right)-2 \mathrm{E}-12\left(X^{5}\right)+7 \mathrm{E}-10\left(X^{4}\right)-1 \mathrm{E}-$

$07\left(X^{3}\right)+6 \mathrm{E}-06\left(X^{2}\right)-0.0003(X)+0.023$

$Y_{2}=-2 \mathrm{E}-15\left(X^{6}\right)+2 \mathrm{E}-12\left(X^{5}\right)-7 \mathrm{E}-10\left(X^{4}\right)+1 \mathrm{E}-$

$07\left(X^{3}\right)-2 \mathrm{E}-06\left(X^{2}\right)-0.0005(X)+0.0015$;

Equations for wind floe $0.7 \mathrm{~m} / \mathrm{s}$ (Equation Set 5):

$Y_{1}=3 \mathrm{E}-15\left(X^{6}\right)-3 \mathrm{E}-12\left(X^{5}\right)+1 \mathrm{E}-09\left(X^{4}\right)-2 \mathrm{E}-$

$07\left(X^{3}\right)+7 \mathrm{E}-06\left(X^{2}\right)-0.0003(X)+0.0263$;

$Y_{2}=-2 \mathrm{E}-15\left(X^{6}\right)+2 \mathrm{E}-12\left(X^{5}\right)-1 \mathrm{E}-09\left(X^{4}\right)+2 \mathrm{E}-$

$07\left(X^{3}\right)-1 \mathrm{E}-05\left(X^{2}\right)-0.0002(X)-0.0012$;

Equations for wind floe $0.8 \mathrm{~m} / \mathrm{s}$ (Equation Set 6):

$Y_{1}=6 \mathrm{E}-15\left(X^{6}\right)-7 \mathrm{E}-12\left(X^{5}\right)+3 \mathrm{E}-09\left(X^{4}\right)-5 \mathrm{E}-$

$07\left(X^{3}\right)+4 \mathrm{E}-05\left(X^{2}\right)-0.0014(X)+0.0392$;

$Y_{2}=-3 \mathrm{E}-15\left(X^{6}\right)+3 \mathrm{E}-12\left(X^{5}\right)-1 \mathrm{E}-09\left(X^{4}\right)+2 \mathrm{E}-$

$07\left(X^{3}\right)-8 \mathrm{E}-06\left(X^{2}\right)-0.0005(X)-0.0122$;

Equations for wind floe $0.9 \mathrm{~m} / \mathrm{s}$ (Equation Set 7):

$Y_{1}=7 \mathrm{E}-15\left(X^{6}\right)-8 \mathrm{E}-12\left(X^{5}\right)+3 \mathrm{E}-09\left(X^{4}\right)-4 \mathrm{E}-$

$07\left(X^{3}\right)+2 \mathrm{E}-05\left(X^{2}\right)-0.0006(X)+0.0339$;

$Y_{2}=-2 \mathrm{E}-15\left(X^{6}\right)+2 \mathrm{E}-12\left(X^{5}\right)-9 \mathrm{E}-10\left(X^{4}\right)+1 \mathrm{E}-$

$07\left(X^{3}\right)+7 \mathrm{E}-06\left(X^{2}\right)-0.0014(X)-0.0046$;

Equations for wind floe 1.0m/s (Equation Set 8): 
$Y_{1}=8 \mathrm{E}-15\left(X^{6}\right)-8 \mathrm{E}-12\left(X^{5}\right)+3 \mathrm{E}-09\left(X^{4}\right)-5 \mathrm{E}-$

$07\left(X^{3}\right)+3 \mathrm{E}-05\left(X^{2}\right)-0.001(X)+0.0482$;

$Y_{2}=-4 \mathrm{E}-15\left(X^{6}\right)+4 \mathrm{E}-12\left(X^{5}\right)-2 \mathrm{E}-09\left(X^{4}\right)+3 \mathrm{E}-$

$07\left(X^{3}\right)-8 \mathrm{E}-06\left(X^{2}\right)-0.0009(X)-0.0175$; 